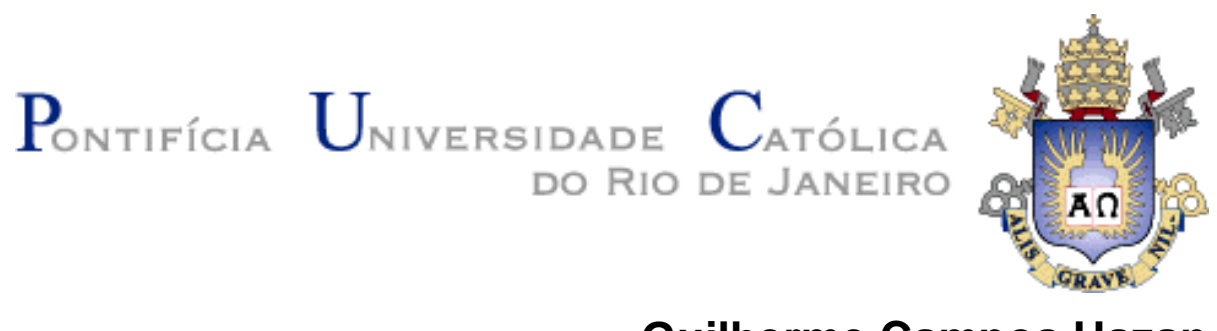

Guilherme Campos Hazan

\title{
Uma Especificação de Máquina de Registradores para Java
}

\section{Dissertação de Mestrado}

Dissertação apresentada como requisito parcial para obtenção do título de Mestre pelo Programa de PósGraduação em Informática da PUC-Rio.

Orientador: Roberto lerusalimschy 


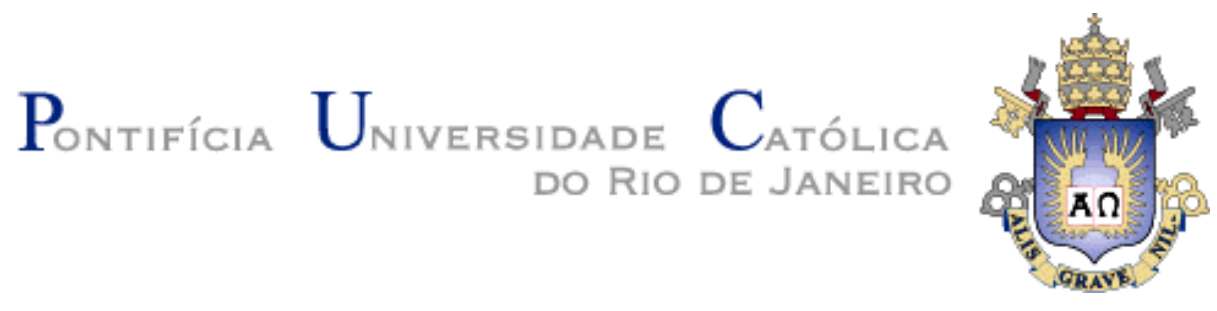

Guilherme Campos Hazan

\title{
Uma Especificação de Máquina de Registradores para Java
}

Dissertação apresentada como requisito parcial para a obtenção do grau de Mestre pelo Programa de Pós-graduação em Informática do Departamento de Informática do Centro Técnico e Científico da PUCRio. Aprovada pela Comissão Examinadora abaixo assinada.

\author{
Prof. Roberto Ierusalimschy \\ Orien- \\ tador \\ Departamento de Informática - PUC- \\ Rio \\ Profa. Noemi de La Rocque Rodriguez \\ Departamento de Informática - PUC- \\ Rio \\ Prof. Renato Fontoura de Gusmão Cerqueira \\ Departamento de Informática - PUC- \\ Rio \\ Prof. José Eugenio Leal \\ Coordenador Setorial do Centro \\ Técnico Científico - PUC-Rio
}

Rio de janeiro, 09 de abril de 2007 
Todos os direitos resenvados. É proibida a reprodução total ou parcial do trabalho sem autorização da universidade, do autor e do orientador.

\section{Guilherme Campos Hazan}

Bacharel em Ciência da Computação pela Universidade Federal do Rio de Janeiro (UFRJ). Começou a programar em 1984. Iniciou em Java em 1998, trabalhando para a Quality Software, onde criou applets de geração de diversos tipos de gráficos, além de uma applet de CAD (2o lugar no Concurso Anual de Comércio Eletrônico da Siemens de 2002). Palestrante em diversas conferências: Comdex Sucesu 2002, One Day Java 2002, Just Java 2003, Java In Rio 2003, Congresso Catarinense de Software Livre 2003, dentre outros. Guilherme é o criador e da máquina virtual SuperWaba. Professor de Java para PDAs na pós-graduação da CESUMAR.

Ficha Catalográfica

Hazan, Guilherme Campos

Uma especificação de máquina de registradores para Java / Guilherme Campos Hazan; orientador: Roberto lerusalimschy. -2007.

72 f. ; $30 \mathrm{~cm}$

Dissertação (Mestrado em Informática)-Pontifícia Universidade Católica do Rio de Janeiro, Rio de Janeiro, 2007. Inclui bibliografia

1. Informática - Teses. 2. Java. 3. Bytecode. 4. Compilador. 5. Máquina virtual. 6. Registrador. 7. Dispositivos móveis. I. lerusalimschy, Roberto. II. Pontifícia Universidade Católica do Rio de Janeiro. Departamento de Informática. III. Título.

Incluí referências bibliográficas.

Java, bytecode, compilador, máquina virtual, registrador, pilha, desempenho, otimização, pda, dispositivos móveis

CDD: 004 
Gostaria de dedicar essa dissertação à Verinha, minha mãe, que faleceu em Fevereiro de 2005. Ela, como Diretora da Escola de Musica da Universidade Federal de Minas Gerais, e Diretora Artística da Fundação Clovis Salgado, foi um exemplo para mim, pelo seu profissionalismo reconhecido pelos colegas de trabaIho e capacidade de tornar um sucesso os trabalhos que fez pela grande dedicação e, principalmente, pela paixão com que tratava a sua profissão. 


\section{Agradecimentos}

Gostaria de agradecer ao meu orientador, Roberto, pelo seu desprendimento em me orientar em um trabalho diferente do que costuma realizar, na plataforma Lua. Isso causava surpresa em todas as pessoas com quem eu conversava sobre o assunto da dissertação e sobre quem era o orientador. Elas indagavam: "... mas nao é sobre Lua?".

Gostaria de agradecer também ao Prof. Arndt von Staa, cuja matéria sobre Testes e Métricas de Software foi de fundamental importância para auxiliar a correta implementação da máquina virtual. 


\section{Resumo}

Hazan, Guilherme Campos; Ierusalimschy, Roberto. Uma Especificação de Máquina de Registradores para Java. PUC-Rio, 2007. 72p. Dissertação de Mestrado - Departamento de Informática, Pontifícia Universidade Católica do Rio de Janeiro.

A linguagem Java foi definida tendo como foco a portabilidade. O código gerado pela compilação é interpretado por uma máquina virtual, e não diretamente pelo processador destino, como um programa em $\mathrm{C}$. Este código intermediário, também conhecido como bytecode, é a chave da portabilidade de Java. Os Bytecodes Java usam uma pilha para manipular os operandos das instruções. O uso de pilha tem suas vantagens e desvantagens. Dentre as vantagens, podemos citar a simplicidade da implementação do compilador e da máquina virtual. A principal desvantagem é a redução na velocidade de execução dos programas, devido à necessidade de se mover os operandos para a pilha e retirar dela o resultado, gerando um aumento no número de instruções que devem ser processadas. Diversos estudos indicam que máquinas virtuais baseadas em registradores podem ser mais rápidas que as baseadas em pilha. Decidimos criar uma nova especificação de bytecodes, específicos para máquinas virtuais baseadas em registradores. Esperamos com isso obter um aumento no desempenho das aplicações.

\section{Palavras-chave}

Java; bytecode; compilador; máquina virtual; registrador; pilha; desempenho; otimização; pda; dispositivos móveis 


\section{Abstract}

Hazan, Guilherme Campos; Ierusalimschy, Roberto. A Specification for a Java Register-Based Machine. PUC-Rio, 2007. 72p. MSc Dissertation Departamento de Informática, Pontifícia Universidade Católica do Rio de Janeiro.

The Java language was created with a focus on portability. The code generated by the compiler is interpreted by a virtual machine, and not directly by the target processor, like programs written in $\mathrm{C}$. This intermediate code, also known as bytecode, is the key to Java's portability. The Java Bytecodes use a stack to manipulate the instruction operands. The use of stack has its their pros and cons. Among the advantages, we can cite the simplicity of implementation of the compiler and virtual machine. On the other hand, there is a speed reduction in the program's execution, due to the need to move the operands to and from the stack, and retrieve results from it, increasing the number of instructions that are processed. Much study has been done that indicating that register-based virtual machines can be faster than the ones based on stacks. Based on this, we decided to create a new bytecode specification, proper for a virtual machine based on registers. By doing this, we hope to obtain an increase in an application's performance.

\section{Keywords}

Java; bytecode; compiler; virtual machine; register; stack; performance; optimization; pda; mobile devices 


\section{Sumário}

1 Introdução

2 Referencial Teórico $\quad 15$

2.1. Tamanho do código armazenado 17

2.2. Desempenho dos programas 18

2.3. Máquinas virtuais baseadas em registradores 20

2.4. Conversão de pilha para registradores 21

3 Nova Arquitetura de Bytecodes 23

3.1. Separação dos registradores em grupos 23

3.2. Quantidade de registradores 24

3.3. Operações com campos 25

3.4. Ortogonalidade das operações 25

3.5. Otimização da tabela de constantes 27

3.6. Arquitetura de palavra das instruções $\quad 27$

3.7. Formato das instruções $\quad 28$

3.8. Escolha das instruções 28

3.9. Listagem das instruções $\quad 37$

4 Avaliação do Código Gerado 46

4.1. Descrição dos testes 46

4.2. Desempenho obtido nas plataformas $\quad 50$

4.3. Tamanho do código gerado 54

5 Conclusão 56

6 Referências Bibliográficas $\quad 59$

7 Apêndice $\quad 61$

7.1. Lista dos bytecodes Java 62

7.2. Mapeamento entre os bytecodes Java e as novas instruções 63 


\section{Lista de tabelas}

Tabela 1: Categorias dos bytecodes Java 16

Tabela 2: Tipos Java 16

Tabela 3: Percentagem da freqüência dinâmica para grupos de Bytecodes 19

Tabela 4: Bytecodes necessários para somar 1 a um campo de instância 19

Tabela 5: Percentagem do número de parâmetros usados em métodos 24

Tabela 6: Percentagem do número de locais usados em métodos 24

Tabela 7: Programas usados durante o teste e resumo dos resultados 32

Tabela 8: Instruções aritméticas e lógicas dos programas Java 33

Tabela 9: Instruções aritméticas e lógicas do J2SE 5.033

Tabela 10: Operandos das instruções aritméticas e lógicas para Java 34

Tabela 11: Operandos das instruções aritméticas e lógicas para J2SE $5.0 \quad 34$

Tabela 12: Instruções de desvio condicional para Java 35

Tabela 13: Instruções de desvio condicional para J2SE $5.0 \quad 36$

Tabela 14: Operandos das instruções de desvio condicional para Java 36

Tabela 15: Operandos das instruções de desvio condicional para J2SE $5.0 \quad 37$

Tabela 16: Número de locais por método para programas Java 37

Tabela 17: Número de locais por método para J2SE 5.0

Tabela 18: Operandos usados nas instruções 40

Tabela 19: Instruções de movimentação 41

Tabela 20: Instruções aritméticas e lógicas $\quad 42$

Tabela 21: Instruções de desvio condicional 43

Tabela 22: Instruções de chamada de método 44

Tabela 23: Instruções de conversão entre tipos de dados 44

Tabela 24: Instruções para retorno de método 44

Tabela 25: Instruções não categorizadas $\quad 45$

Tabela 26: Partes que diferem entre as plataformas $\quad 48$

Tabela 27: Partes comuns às plataformas $\quad 48$

Tabela 28: Código fonte dos testes realizados no Pentium 4-M. 49

Tabela 29: Tempos em milisegundos dos testes realizados no Pentium 4-M 53

Tabela 30: Número de instruções dentro do laço 53

Tabela 31: Tempos em milisegundos dos testes realizados no Dell Axim X3 53

Tabela 32: Bytecodes Java 62 


\section{Lista de figuras}

Figura 1: Composição de um arquivo class 18

$\begin{array}{lr}\text { Figura 2: Tabela de constantes } & 18\end{array}$

Figura 3: Comparação dos tipos primitivos em relação ao int. 26

Figura 4: Código usado para medir o tempo de execução 26

Figura 5: Gráfico comparativo com os tempos obtidos no Pentium 4-M 52

Figura 6: Gráfico comparativo com os tempos obtidos no Dell Axim X3 52

Figura 7: Tamanho do código gerado pela compilação do teste 55

Figura 8: Composição do arquivo gerado pela compilação do teste 55

Figura 9: Tamanho do código gerado pela compilação dos protótipos 55 